Limit Pricing and Entry under Incomplete Information: An Equilibrium Analysis Author(s): Paul Milgrom and John Roberts

Source: Econometrica, Vol. 50, No. 2 (Mar., 1982), pp. 443-459

Published by: The Econometric Society

Stable URL: http://www.jstor.org/stable/1912637

Accessed: 07/12/2010 13:14

Your use of the JSTOR archive indicates your acceptance of JSTOR's Terms and Conditions of Use, available at http://www.jstor.org/page/info/about/policies/terms.jsp. JSTOR's Terms and Conditions of Use provides, in part, that unless you have obtained prior permission, you may not download an entire issue of a journal or multiple copies of articles, and you may use content in the JSTOR archive only for your personal, non-commercial use.

Please contact the publisher regarding any further use of this work. Publisher contact information may be obtained at http://www.jstor.org/action/showPublisher?publisherCode=econosoc.

Each copy of any part of a JSTOR transmission must contain the same copyright notice that appears on the screen or printed page of such transmission.

JSTOR is a not-for-profit service that helps scholars, researchers, and students discover, use, and build upon a wide range of content in a trusted digital archive. We use information technology and tools to increase productivity and facilitate new forms of scholarship. For more information about JSTOR, please contact support@jstor.org. 


\title{
LIMIT PRICING AND ENTRY UNDER INCOMPLETE INFORMATION: AN EQUILIBRIUM ANALYSIS ${ }^{1}$
}

\author{
By Paul Milgrom and John Roberts
}

\begin{abstract}
Limit pricing involves charging prices below the monopoly price to make new entry appear unattractive. If the entrant is a rational decision maker with complete information, pre-entry prices will not influence its entry decision, so the established firm has no incentive to practice limit pricing. However, if the established firm has private, payoff relevant information (e.g., about costs), then prices can signal that information, so limit pricing can arise in equilibrium. The probability that entry actually occurs in such an equilibrium, however, can be lower, the same, or even higher than in a regime of complete information (where no limit pricing would occur).
\end{abstract}

\section{INTRODUCTION}

THE BASIC IDEA OF LIMIT PRICING is that an established firm may be able to influence, through its current pricing policy alone ${ }^{2}$ other firms' perceptions of the profitability of entering the firm's markets, and that the firm may thus set its prices below their short run maximizing levels in order to deter entry. As such, limit pricing has constituted a major theme in the industrial organization literature for at least the last thirty years, and during the past decade in particular it has been the subject of a number of papers employing formal models of maximizing behavior. ${ }^{3}$ For the most part, these latter analyses have concentrated on the decision problem of the established firm, taking as given the limit-pricing assumption that a lower pre-entry price will deter or restrict entry. In this context, the typical conclusion is that an optimal price-output policy in the face of threatened entry will involve prices which are below the short-run monopoly level, but still above the level that would prevail after entry. This conclusion had led to some debate as to the appropriate public policy regarding such limit pricing, since there appears to be a trade-off between the benefits to society of lower pre-entry prices and the costs arising from entry being limited or deterred.

\footnotetext{
${ }^{1}$ Much of the work reported here first appeared in [11]. This work has been presented at a large number of conferences, meetings, and seminars, and we would like to thank our audiences at each of these events for their comments. We are particularly indebted to Eric Maskin, Roger Myerson, Steve Salop, Robert Wilson, and two referees for their helpful suggestions, to David Besanko for his excellent research assistance, and to Armando Ortega-Reichert, whose work on repeated competitive bidding [15] has influenced our thinking on the present subject. Finally, we gratefully acknowledge the financial support of the Graduate School of Business at Stanford, the J. L. Kellogg Graduate School of Management at Northwestern, and the National Science Foundation (Grants SOC 77-06000 to the IMSSS at Stanford and SOC 79-07542 and SES 80-01932 to Northwestern).

${ }^{2}$ Although some recent treatments of entry deterrence incorporate other strategic variables, the standard, traditional approach is to treat the choice of the pre-entry price as the firm's only decision and to assume no dependence of post-entry profits on this choice.

${ }^{3}$ The idea behind limit pricing can be traced back through the work of J. Bain [1] and J. M. Clark [2] at least to a paper by N. Kaldor [7]. The recent formal investigations begin with D. Gaskins [5], M. I. Kamien and N. L. Schwartz [8], and G. Pyatt [16]. See F. M. Scherer [18] and S. Salop [17] for further references.
} 
In this paper we present a re-examination of the limit pricing problem. Our model differs from most of the existing literature in that we treat both the established firm and potential entrant as rational, maximizing economic agents. This naturally leads to a game-theoretic, equilibrium formulation. However, once one adopts this approach, it is not immediately obvious why limit pricing should emerge at all.

This point has been made explicitly by J. Friedman [3] in one of the few existing game-theoretic treatments of pricing in the face of potential entry of which we are aware. Friedman notes that, under the usual sort of assumptions on demand, the profits which would accrue should entry occur are completely independent of the pre-entry price. Since in Friedman's model both the established firm and the entrant are completely informed as to demand and cost conditions, these post-entry profits are fully known when the entry decision is made. Then the inescapable logic of (perfect) equilibrium (Selten [19]) requires that the entry decision be independent of the pre-entry price. This means that any attempt at limit pricing would serve only to squander pre-entry profits and so there would be no limit pricing.

Friedman's argument will be generally valid in any complete-information, game-theoretic model in which the established firm's pre-entry actions do not influence post-entry costs and demand. In such a model, then, the intuitive idea underlying the traditional concept of limit pricing - that potential entrants would read the pre-entry price as a signal concerning the price and market shares they can expect to prevail after entry - finds no formal justification. In contrast, a formalization of this intuition is the very heart of our model.

Specifically, we consider situations in which neither the established firm nor the potential entrant is perfectly informed as to some characteristic of the other which is relevant to the post-entry profits of both. The central example of such a characteristic, and the one on which we initially concentrate, is the other firm's unit costs. In such a situation, the pre-entry price may become a signal regarding the established firm's costs, which in turn are a determinant of the post-entry price and profits for the entrant. Thus the relationship assumed in the earlier literature emerges endogenously in equilibrium in our model: a lower price (by signalling lower costs) tends to discourage entry. Thus, too, limit-pricing behavior arises in equilibrium, with the established firm attempting to influence the entry decision by charging a pre-entry price which is below the simple monopoly level.

The entrant, meanwhile, will seek to infer the established firm's costs (and thus the profitability of entry) from observation of the pre-entry price. In making this inference, of course, it will have to employ some conjecture regarding the established firm's pricing policy, i.e., the relationship between the established firm's cost and the price it charges. In Nash equilibrium, this conjecture must be correct. Indeed the very definition of equilibrium in this context involves rational expectations by each firm about the other's behavior. Thus, the entrant will allow for limit pricing in making its inferences and its entry decision.

Thus, in equilibrium, the established firm practices limit pricing, but the 
entrant is not fooled by this strategy. Consequently, the probability that entry actually occurs in equilibrium need not be any lower than it would be in a world of full information, where limit pricing would not arise. Indeed, the probability of entry in the limit pricing equilibrium may even be higher than with complete information, even though the pre-entry price is lower. In particular, this means that the alleged trade-off for society between lower prices and delayed or deterred entry may never arise.

In the next section, we illustrate these claims in the context of a simple model with linear demand and constant unit costs. In this model we compute equilibria for two specific examples. One of these involves only two possible levels of costs for the entrants and for the established firm; the other involves a continuum of possibilities on each side. In Section 3 we consider a more general model. The final section contains our conclusions.

\section{TWO EXAMPLES}

Consider the market for a homogeneous good in which there is an established firm, denoted firm 1, and a potential entrant, firm 2. Initially, each firm knows its own unit cost, $c_{i}, i=1,2$, but it does not know the other firm's cost level. Firm 1 is a monopolist, and it must pick a quantity $Q$ to produce (or a price to charge) as a monopolist, given its knowledge of $c_{1}$ and its beliefs about $c_{2}$. Firm 2 will observe this choice and then (knowing $c_{2}$ but not $c_{1}$ ) must either enter the market or decide to stay out. If it enters, it incurs an entry cost of $K$, each firm learns the other's cost, and then the two firms operate as Cournot duopolists. If it does not enter, firm 1 will henceforth enjoy its monopoly profits without further fear of entry.

We summarize the notation and profit formulae with linear demand and constant unit costs in Table I. To simplify the payoff formulae, we normalize the

\section{TABLE I}

$\begin{array}{lr}\text { Present value to } i \text { of } \$ 1 \text { accruing after entry } & \delta_{i} \\ \text { Unit production cost of firm } i & c_{i} \\ \text { Fixed cost of entry for firm 2 } & K \\ \text { Inverse demand } & m\left(c_{1}\right)=\left(a-c_{1}\right) / 2 b \\ \text { Simple monopoly output } & \Pi_{1}^{0}\left(Q, c_{1}\right)=\left(a-b Q-c_{1}\right) Q \\ \text { First period profit for firm 1 } & \Pi_{1}^{M}\left(c_{1}\right)=\left(a-c_{1}\right)^{2} / 4 b \\ \text { Monopoly profit for firm 1 } & \Pi_{i}^{c}\left(c_{1}, c_{2}\right)=\left(a-2 c_{i}+c_{j}\right)^{2} / 9 b \\ \text { Cournot profit for firm } i & R\left(c_{1}, c_{2}\right)=\Pi_{1}^{M}\left(c_{1}\right)-\Pi_{1}^{C}\left(c_{1}, c_{2}\right) \\ \text { Reward to firm 1 from deterring entry } & \Pi_{1}^{O}\left(Q, c_{1}\right) \\ \text { Payoff to } 1 \text { if entry occurs } & \Pi_{1}^{0}\left(Q, c_{1}\right)+\delta_{1} R\left(c_{1}, c_{2}\right) \\ \text { Payoff to } 1 \text { if no entry } & \delta_{2} \Pi_{2}^{C}\left(c_{1}, c_{2}\right)-K \\ \text { Payoff to } 2 \text { if entry } & 0 \\ \text { Payoff to } 2 \text { if no entry } & {\left[c_{i}, \bar{c}_{i}\right]} \\ \text { Range of possible } c_{i} \text { values } & \\ \text { Probability distribution function for } c_{i} & H_{i} \\ \quad\left(j \text { 's beliefs about } c_{i}\right) & \end{array}$


post-entry profits of the established firm to be zero if entry occurs, so it receives only its first period profit as its payoff in this event. If entry does not occur, its payoff is its first period profit plus the discounted value of a reward to deterring entry. This reward is equal to the excess of its monopoly profit over its profit as a Cournot duopolist.

The extensive form game corresponding to this set-up is one of incomplete information, since the players do not know the numerical values of the payoffs corresponding to any pair of decisions they make. Attempting to analyze such a game directly would easily lead one into a morass of infinite regress. The approach we adopt instead is that proposed by Harsanyi [6], which involves replacing this incomplete information game by a game of complete but imperfect information. ${ }^{4}$ One then treats the Nash equilibria of this second game as the equilibria of the original game.

The imperfect information game involves another player, "Nature," which is indifferent over all possible outcomes. Nature moves first and selects $c_{1}$ and $c_{2}$ according to the probability distributions, $H_{i}$, giving the players' beliefs. Then player $i$ is informed about $c_{i}$ but not about $c_{j}$, and for each realization of $c_{1}$ and $c_{2}$ the game tree unfolds as above.

In any extensive form game, a player's strategy is a specification of the action it will take in any information set, i.e., the player's actions at any point can depend only on what it knows at that point. Here, the information sets for firm 1 are defined by the realized values of $c_{1}$ (given by "Nature's move") and those for firm 2 by a realization of $c_{2}$ and a choice of $Q$ by firm 1 . Thus, a (pure) strategy for 1 is a map $s$ from its possible cost levels into the possible choices of $Q$ and a (pure) strategy for 2 is a map $t$ from $\mathbb{R}^{2}$ into $\{0,1\}$ giving its decision for each possible pair $\left(c_{2}, Q\right)$, where we interpret 1 as "enter" and 0 as "stay out."

A pair of strategies constitutes an equilibrium if each maximizes the expected payoff of the player using it, given that the other is using its specified strategy. This is the standard Nash equilibrium notion. However, to accentuate the rational expectations character of Nash equilibrium, it is helpful to use the following, equivalent definition. An equilibrium consists of a pair of strategies $\left(s^{*}, t^{*}\right)$ and a pair of conjectures $(\bar{s}, \bar{t})$ such that (i) firm l's pricing policy $s^{*}$ is a best response to its conjecture $\bar{t}$ about firm 2's entry rule, (ii) the strategy $t^{*}$ is a best response for firm 2 to its conjecture $\bar{s}$, and (iii) the actual and conjectured strategies coincide. We formalize these conditions as follows: (i) for any $c_{1} \in$ $\left[\underline{c}_{1}, \bar{c}_{1}\right]$ and any $s:\left[\underline{c}_{1}, \bar{c}_{1}\right] \rightarrow \mathbb{R}_{+}$,

$$
\begin{aligned}
& \Pi^{0}\left(s^{*}\left(c_{1}\right), c_{1}\right)+\delta_{1} \int_{\underline{c}_{2}}^{\bar{c}_{2}} R\left(c_{1}, c_{2}\right)\left[1-\bar{t}\left(c_{2}, s^{*}\left(c_{1}\right)\right)\right] d H_{2}\left(c_{2}\right) \\
& \geqq \Pi^{0}\left(s\left(c_{1}\right), c_{1}\right)+\delta_{1} \int_{\underline{c}_{2}}^{\bar{c}_{2}} R\left(c_{1}, c_{2}\right)\left[1-\bar{t}\left(c_{2}, s\left(c_{1}\right)\right)\right] d H_{2}\left(c_{2}\right),
\end{aligned}
$$

\footnotetext{
${ }^{4}$ An extensive form game has imperfect information if some player at some point must make a move without having been fully informed about all the previous moves made by the other players.
} 
(ii) for any $c_{2} \in\left[\underline{c}_{2}, \bar{c}_{2}\right]$ and any $t:\left[\underline{c}_{2}, \bar{c}_{2}\right] \times \mathbb{R}_{+} \rightarrow\{0,1\}$,

$$
\begin{aligned}
\int_{\underline{c}_{1}}^{\bar{c}_{1}}[ & \left.\delta_{2} \Pi_{2}^{C}\left(c_{1}, c_{2}\right)-K\right] t^{*}\left(c_{2}, \bar{s}\left(c_{1}\right)\right) d H_{1}\left(c_{1}\right) \\
& \geqq \int_{\underline{c}_{1}}^{\bar{c}_{1}}\left[\delta_{2} \Pi_{2}^{C}\left(c_{1}, c_{2}\right)-K\right] t\left(c_{2}, \bar{s}\left(c_{1}\right)\right) d H_{1}\left(c_{1}\right), \quad \text { and }
\end{aligned}
$$

(iii) $\left(s^{*}, t^{*}\right)=(\bar{s}, \bar{t})$.

Given this framework, we first study a parameterized family of examples where the $H_{i}$ are two-point distributions and, for specific values of the parameters, compute equilibria. Later in this section we will allow for a continuum of possible cost levels ("types") for the two firms.

Thus, suppose that the demand curve is $P=10-Q$, that $K=7$, that $\underline{c}_{1}=0.5$, $\underline{c}_{2}=1.5, \bar{c}_{1}=\bar{c}_{2}=2.0$, that $\delta_{1}=\delta_{2}=1$, and that the costs are independently distributed with $H_{2}\left(c_{2}=\bar{c}_{2}\right)=p=1-H_{2}\left(c_{2}=\underline{c}_{2}\right)$ and $H_{1}\left(c_{1}=\bar{c}_{1}\right)=q=1-$ $H_{1}\left(c_{1}=\underline{c}_{1}\right)$.

With these specifications, the payoffs are as follows:

$$
\begin{aligned}
R\left(\underline{c}_{1}, \underline{c}_{2}\right)=10.31, & \Pi_{2}^{C}\left(\underline{c}_{1}, \underline{c}_{2}\right)-K=-0.75, \\
R\left(\underline{c}_{1}, \bar{c}_{2}\right)=9.12, & \Pi_{2}^{C}\left(\underline{c}_{1}, \bar{c}^{2}\right)-K=-2.31, \\
R\left(\bar{c}_{1}, \underline{c}_{2}\right)=9.75, & \Pi_{2}^{C}\left(\bar{c}_{1}, \underline{c}_{2}\right)-K=2.00, \\
R\left(\bar{c}_{1}, \bar{c}_{2}\right)=8.89, & \Pi_{2}^{C}\left(\bar{c}_{1}, \bar{c}_{2}\right)-K=0.11, \\
m\left(\underline{c}_{1}\right)=4.75, & \Pi_{1}^{M}\left(\underline{c}_{1}\right)=22.56, \\
m\left(\bar{c}_{1}\right)=4.00, & \Pi_{1}^{M}\left(\bar{c}_{1}\right)=16.00 .
\end{aligned}
$$

Note that if l's costs were known to be $\underline{c}_{1}$, neither type of potential entrant would want to enter, while if $c_{1}$ were known to be $\bar{c}_{1}$, both would want to enter. Thus, the probability of entry, if the entrant were to be directly informed of the realized value of $c_{1}$, is simply $q$, the probability that $c_{1}=\bar{c}_{1}$. Of course, if firm 2 were so informed, there would be no point to limit pricing and $Q$ would simply be set at the short-run profit-maximizing level of $m\left(c_{1}\right)$.

Note, too, that if firm 2 were unable to observe $Q$ and were uninformed about $c_{1}$, then it would want to enter if its expected profits were positive, i.e., if $q \Pi_{2}^{C}\left(\bar{c}_{1}, c_{2}\right)+(1-q) \Pi_{2}^{C}\left(\underline{c}_{1}, c_{2}\right)-K \geqq 0$. If $0.954>q>0.273$, then this inequality holds for $\underline{c}_{2}$ and not for $\bar{c}_{2}$, so the low cost entrant would come in and the high cost entrant would not. (For $q<0.273$, neither would want to enter, and for $q>0.954$, both would want to enter.)

In fact, if 2 is not directly informed about $c_{1}$ but can observe $Q$, it will attempt to make inferences about the actual value of $c_{1}$ from its observation of $Q$, using its conjectures about 1's behavior. Note that in equilibrium, the only values of $Q$ which could be observed are $s^{*}\left(\underline{c}_{1}\right)$ and $s^{*}\left(\bar{c}_{1}\right)$. Now in this set-up there are only two possibilities: either $s^{*}\left(\underline{c}_{1}\right)=s^{*}\left(\bar{c}_{1}\right)$, or else the two values differ. An equilibrium with the first of these properties is called pooling, while in the other situation 
the equilibrium is separating. Thus, in pooling equilibrium, observing $Q$ gives no information, while the observation of $Q$ in a separating equilibrium allows the value of $c_{1}$ to be inferred exactly.

Thus, in a separating equilibrium $\left(s^{*}, t^{*}\right)$, entry will occur if $s^{*}\left(\bar{c}_{1}\right)$ is observed and will not if $s^{*}\left(\underline{c}_{1}\right)$ is observed: entry takes place in exactly the same circumstances as if the entrant had been informed about the value of $c_{1}$, i.e., with prior probability $q$. Moreover, this will be true in any separating equilibrium of any model of this type: in any separating equilibrium, observing the equilibrium choice of the established firm allows a precise and accurate inference to be made about the firm's characteristic. Thus, in such an equilibrium, limit pricing will not limit entry relative to the complete information case (in which there would be no limit pricing because the possibility of influencing the entrant's decision does not arise).

In a pooling equilibrium, the entrant can infer nothing from observing $Q$ and so enters if its expected profit is positive. Thus, as noted above, if $q \in(0.273$, 0.954 ), only the low cost entrant will come in. Thus, in a pooling equilibrium, the probability of entry is $(1-p)$, while in a separating equilibrium the probability of entry is $q$.

We now will show that, in this example, so long as $p$ is not too small, there are both pooling and separating equilibria, that all equilibria involve limit pricing, and that the probability of entry in a pooling equilibrium may equal, exceed or fall short of that in a separating equilibrium (or, equivalently, under complete information).

First, we show that the following strategies constitute a separating equilibrium:

$$
\begin{aligned}
& s^{*}\left(\underline{c}_{1}\right)=7.2, \quad s^{*}\left(\bar{c}_{1}\right)=m\left(\bar{c}_{1}\right)=4.0, \\
& t^{*}\left(c_{2}, Q\right)= \begin{cases}1 & \text { if } Q<7.2, \\
0 & \text { otherwise }\end{cases}
\end{aligned}
$$

Note that since $s^{*}\left(\underline{c}_{1}\right)>m\left(\underline{c}_{1}\right), s^{*}$ is a limit pricing strategy. Notice too that from our earlier discussion, $t^{*}$ is clearly a best response to $s^{*}$. Thus, we need to check that $s^{*}$ is optimal, given $t^{*}$. First, note that unless the high cost established firm produces at least 7.2 , it cannot deter any entry. But, this level is high enough that it is not worthwhile for $\bar{c}_{1}$ to produce it, even though in so doing it would eliminate all entry. To see this, note that producing $Q=s^{*}\left(\underline{c}_{1}\right)$ yields the payoff

$$
\Pi_{1}^{0}\left(\bar{c}_{1}, s^{*}\left(\underline{c}_{1}\right)\right)+p R\left(\bar{c}_{1}, \bar{c}_{2}\right)+(1-p) R\left(\bar{c}_{1}, \underline{c}_{2}\right)=15.51-0.86 p
$$

while producing $m\left(\bar{c}_{1}\right)$ yields $\Pi_{1}^{0}\left(\bar{c}_{1}, m\left(\bar{c}_{1}\right)\right)=16$, which exceeds $15.51-0.86 p$ for all $p \geqq 0$. Finally, note that the low cost firm has no reason to produce more than $s^{*}\left(\underline{c}_{1}\right)$. If it produces less, it is sure to face entry, and thus its best choice in this range would be $m\left(\underline{c}_{1}\right)$. But $s^{*}\left(\underline{c}_{1}\right)$ yields an expected payoff of $26.87-1.19 p$, which for all $p \leqq 1$ strictly exceeds the payoff $\Pi_{1}^{M}\left(\underline{c}_{1}\right)=22.56$ from producing $m\left(\underline{c}_{1}\right)$. Thus, $s^{*}\left(\underline{c}_{1}\right)$ is also optimal. 
We now demonstrate the existence of a pooling equilibrium given by

$$
\begin{aligned}
& s^{*}\left(\underline{c}_{1}\right)=s^{*}\left(\bar{c}_{1}\right)=m\left(\underline{c}_{1}\right)=4.75, \\
& t^{*}\left(\underline{c}_{2}, Q\right)=1, \\
& t^{*}\left(\bar{c}_{2}, Q\right)= \begin{cases}0 & \text { if } Q \geqq 4.75, \\
1 & \text { otherwise. }\end{cases}
\end{aligned}
$$

Note again that our earlier discussion indicates that $t^{*}$ is a best response to $s^{*}$, given $q \in(0.273,0.954)$. Further, it is evident that $s^{*}$ is optimal if $c_{1}=\underline{c}_{1}$, since any increase in $Q$ would not deter entry, and any decrease in output would both increase entry and reduce first period profits. Finally, if the established firm has $c_{1}=\bar{c}_{1}$, it similarly has no incentive to increase output, while cutting output could at best yield the monopoly first period return, but would induce certain entry. This gives a payoff of 16.00 , which is, for $p>0.063$, less than its current return of $\Pi_{1}^{0}\left(\bar{c}_{1}, 4.75\right)+p R\left(\bar{c}_{1}, \bar{c}_{2}\right)=15.44+8.89 p$. Thus, if $p>0.063$, this is also an equilibrium, and since $s^{*}\left(\bar{c}_{1}\right)>m\left(\bar{c}_{1}\right)$, it, too, involves limit pricing.

To summarize, our pooling equilibrium required that the probability $p$ of the entrant having high costs exceed 0.063 and that $q$ lie in $(0.273,0.954)$, while our separating equilibrium existed for all $p$ and $q$. In a separating equilibrium, the probability of entry is $q$, which is just the probability that the established firm is of the high cost type, while in our pooling equilibrium, the probability of entry is $1-p$, the probability of the entrant having low costs. ${ }^{5}$ Clearly, we may have $1-p$ greater than, less than, or equal to $q$ and still meet the requirements for existence of both equilibria. Limit pricing equilibria may involve less, the same, or more entry than occurs in the full information (no limit pricing) case.

It is, of course, true in either type of equilibrium that if the limit-pricing firm were to charge a higher price than is called for by the equilibrium strategy, then it would face a greater threat of entry. This is because the entrant would interpret this high price as meaning that the firm's costs were higher than they in fact are, and thus entry would appear more attractive. (Note that the entrant's inferences will be correct only if firm 1 adheres to its equilibrium strategy.) Indeed, it is this balancing of foregone first period profits against the reward to deterring entry which characterizes the equilibrium and it is this threat of increased entry which leads the established firm to maintain its expanded output. Thus, in this sense, limit pricing does limit entry.

A useful way to think about these results is to consider limit pricing as the outcome of competition between the types of the established firm, with high cost types attempting to mimic low cost ones and low cost firms attempting to distinguish themselves from the high cost ones. Then whether a pooling or a

If $q<0.273$, then there is a pooling equilibrium against which the probability of entry is zero. If $q>0.954$, then entry would be certain if a pooling equilibrium were established. But then each type of established firm would find that its monopoly output represents a profitable deviation. Thus, there could be no such equilibrium. 
separating equilibrium is established is a matter of whether it is the high or low cost type which is successful. This competition could, of course, be purely a conjectural one in the mind of the entrant, but it might also be more concrete. Specifically, one can imagine that there are a number of currently monopolized markets, all of which are identical except that a percentage $p$ have high cost incumbents and the rest have low cost incumbents. There is also a limited supply of venture capital, which is available to an entrant whose costs are unknown a priori. Then the competition between types of established firms becomes real, with each established firm attempting to make entry into its market appear unattractive. ${ }^{6}$

The active role assigned to the entrant in this model and the corresponding significance of the beliefs and conjectures embodied in the entrant's strategy lead to the existence of a multiplicity of equilibria, both in this example and more generally. Our example actually has a continuum of both separating and pooling equilibria, where each class of equilibria is parameterized by the critical level of $Q$ such that observation of a lower output than this level induces increased entry. In general, there is a large class of entrant's strategies $t$ such that $t$ and the best response to it constitute an equilibrium: many possible conjectures by the entrant as to the outcome of the competition among established firms are consistent with rational expectations. Thus, there is no unique limit price in these models. ${ }^{7}$

One way to attempt to narrow the set of equilibria is to place restrictions on the possible strategies for the entrant. For example, one could require that, conditional on observing any $Q$, the entrant assign probabilities to $Q$ having been the choice of each type of established firm. Then one would require that, for each $Q, t^{*}\left(c_{2}, Q\right)$ be a best response, given these conjectures. This is the essence of the concept of sequential equilibrium due to David Kreps and Robert Wilson [10], and it is clearly in the spirit of the perfectness criterion for equilibria (Selten [19]). ${ }^{8}$ However, as is easily verified, our equilibria already satisfy this condition, and still we have the unwanted multiplicity. Thus one might consider further restrictions on the entrant's conjectures. In particular, one might hypothesize that the entrant will not conjecture that the competition between types of established firm will be unnecessarily wasteful. This results in considering only those equilibria $\left(s^{*}, t^{*}\right)$ for which there is no other equilibrium where the payoffs to the various types of established firms weakly dominate those under $\left(s^{*}, t^{*}\right)$. The two particular equilibria we have identified here meet this condition. Other separating

\footnotetext{
${ }^{6}$ See E. Gal-or [4] for a more explicit model along these lines. Also see D. Kreps and R. Wilson [9] and P. Milgrom and $\mathbf{J}$. Roberts [12] for multi-market models of entry deterrence through predation.

${ }^{7}$ There is a second source of non-uniqueness which involves the specification of $t^{*}\left(c_{2}, Q\right)$ for values of $Q$ outside the range of $s^{*}$. Since such values of $Q$ are observed with probability zero, the maximization of expected return places no constraint on $t^{*}$ at these points. Then, even within the constraint that $s^{*}$ be a best response to the entrant's strategy, there are typically many strategies $t^{*}$ which constitute equilibria with $s^{*}$. However, all such $t^{*}$ for a given $s^{*}$ give the same evolution of the play of the game (the same $Q$ values being chosen and the same entry decisions being made). Thus, this non-uniqueness is less crucial.

${ }^{8}$ This correspondence is not coincidence, as Kreps and Wilson [10] have shown: every perfect equilibrium is sequential.
} 
equilibria all involve $s^{*}\left(\bar{c}_{1}\right)=m\left(\bar{c}_{1}\right)$ and $s^{*}\left(\underline{c}_{1}\right)>7.2,{ }^{9}$ other pooling equilibria must involve lower payoffs for the low cost established firm, ${ }^{10}$ and neither equilibrium dominates the other.

Although there are no equilibria in this example where $s^{*} \equiv m$, the monopoly output, this strategy could arise in equilibrium with other specifications of the parameters. This would happen if the profit to a high cost firm in producing its monopoly output and then facing certain entry exceeded its profits from producing the monopoly output of the low cost firm and then avoiding all entry. However, if there are a continuum of types (cost levels) possible for the established firm and the $H_{i}$ are atomless, this cannot happen: at most only a set of firms of measure zero could produce their monopoly outputs in equilibrium.

Both to establish this claim and to explore more completely the nature of the limit pricing problem in a framework with less discontinuity, we now examine a specification of the model with a continuum of possible cost levels. Thus, suppose that the distribution of $c_{j}$ is given by a continuous density function $h_{j}\left(c_{j}\right)$ which is positive on $\left[\underline{c}_{j}, \bar{c}_{j}\right]$. We will initially concentrate on separating equilibria.

Assume that 2 conjectures that 1 will play some strategy $\bar{s}$. Then, for any $Q$ in the range of $\bar{s}$, the entrant's best response is to act as if $c_{1} \in \bar{s}^{-1}(Q)$, and to enter if and only if the expected value of $\delta_{2} \Pi_{2}^{C}\left(c_{1}, c_{2}\right)-K$, conditional on $c_{1}$ $\in \bar{s}^{-1}(Q)$, is positive. If $\bar{s}$ is monotone decreasing, then $\bar{s}^{-1}(Q)$ is a singleton and so 2 should enter if and only if $c_{2} \leqq \gamma\left(\bar{s}^{-1}(Q)\right)$, where $\gamma\left(c_{1}\right) \equiv\left(a+c_{1}-\right.$ $3 \sqrt{b K}) / 2$ is the highest level of $c_{2}$ permitting successful entry against a firm with $\operatorname{costs} c_{1}$. Thus, for $Q \in \operatorname{range} \bar{s}$, 2's best response satisfies

$$
t\left(c_{2}, Q\right)= \begin{cases}1 & \text { if } c_{2} \leqq \bar{g}(Q), \\ 0 & \text { otherwise }\end{cases}
$$

where $\bar{g}=\gamma \circ \bar{s}^{-1}$.

Now, suppose that 1's conjecture is that $t$ is of this general form, so that 2 will be deterred from entering if $c_{2}$ exceeds some value $g(Q)$. Then 1's expected payoff is

$$
G\left(c_{1}, Q\right)=\Pi_{1}^{0}\left(c_{1}, Q\right)+\delta_{1} \int_{g(Q)}^{\bar{c}_{2}} R\left(c_{1}, c_{2}\right) h_{2}\left(c_{2}\right) d c_{2} .
$$

Maximizing with respect to $Q$ yields

$$
0=\frac{\partial \Pi_{1}^{0}}{\partial Q}-\delta_{1} R\left(c_{1}, g(Q)\right) h_{2}(g(Q)) g^{\prime}(Q) .
$$

\footnotetext{
${ }^{9}$ Note, in particular, that $s^{*}=m$ is not an equilibrium strategy, since the $\bar{c}_{1}$ firm would be willing to produce $m\left(\underline{c}_{1}\right)$ to eliminate all entry.

${ }^{10}$ While it might seem that any other pooling equilibrium would have $s^{*}\left(c_{1}\right)>m\left(\underline{c}_{1}\right)$, this need not be the case. However, if the entrant's conjectures regarding the value of $c_{1}$, given $Q$, are continuous in $Q$, pooling equilibria with higher than monopoly prices disappear. If, in addition, the probability assigned to $c_{1}=\underline{c}_{1}$ rises sufficiently rapidly in $Q$, then only separating equilibria can exist. These continuity and monotonicity conditions are similar in spirit to Myerson's properness criterion [14].
} 
But, in equilibrium, the conjectures must be correct (i.e., $\bar{s}=s^{*}, g=\gamma \circ s^{*-1}$ ), so we have that $s^{*}\left(c_{1}\right)$ must satisfy

$$
0=\frac{\partial \Pi_{1}^{0}\left(c_{1}, s^{*}\left(c_{1}\right)\right)}{\partial Q}-\frac{\delta_{1} R\left(c_{1}, \gamma\left(c_{1}\right)\right) h_{2}\left(\gamma\left(c_{1}\right)\right) \gamma^{\prime}\left(c_{1}\right)}{d s^{*}\left(c_{1}\right) / d c_{1}}
$$

Note that, so long as $R\left(c_{1}, \gamma\left(c_{1}\right)\right), h_{2}\left(\gamma\left(c_{1}\right)\right)$, and $\gamma^{\prime}\left(c_{1}\right)$ are positive and $d s^{*} / d c_{1}<\infty$ (i.e., $s^{*}$ is differentiable at $c_{1}$ ), then this first order condition implies that $\partial \mathrm{II}_{1}^{0} / \partial Q<0$. Thus, the simple monopoly solution $m\left(c_{1}\right)$, which is defined by $\partial \Pi_{1}^{0} / \partial Q=0$, cannot arise in equilibrium. If the entrant were to conjecture $\bar{s}=m$ and respond optimally, then by increasing output slightly from $m\left(c_{1}\right)$ to, say, $m\left(c_{1}\right)+\epsilon=\bar{s}\left(c_{1}^{\prime}\right)$, the established firm can eliminate the threat of entry from firms in the interval $\left(\gamma\left(c_{1}^{\prime}\right), \gamma\left(c_{1}\right)\right]$. This increase in output has a first-order effect on $\Pi_{1}^{0}$ of zero, since $\partial \Pi_{1}^{0} / \partial Q=0$ at $m\left(c_{1}\right)$, but a non-negligible first-order effect on the expected value of the reward to deterring entry. Thus, in any model of this type, so long as: (i) it is more profitable to be a monopolist than to share the market, (ii) beliefs are given by a positive density, and (iii) higher costs for the established firm encourage entry, essentially all established firms must be limit pricing in a separating equilibrium.

Of course, in such an equilibrium, $s^{*}$ is invertible and so there is the same entry as if $c_{1}$ were known directly.

Now, to obtain an explicit solution for a particular specification, suppose that $\underline{c}_{i}=0$ and that $h_{2}$ has, for $c_{2} \geqq \gamma(0)$, the particular form

$$
h_{2}\left(c_{2}\right)=8 b \rho /\left[4\left(a-c_{2}\right) \sqrt{b K}-7 b K\right]
$$

where the parameter $\rho$ reflects the probability of there being a viable potential competitor. Also, assume that $\bar{c}_{i}<a / 2$, which insures that the usual first-order conditions define a Cournot equilibrium after entry. As well, assume that $a \geqq 7 \sqrt{b K} / 2$, which both insures that $h_{2}$ is a density for any choice of $\bar{c}_{2}<a / 2$ and also implies that $\gamma(0)>0$, so that even low cost established firms are threatened by entry. Finally, assume that $\gamma\left(\bar{c}_{1}\right)<\bar{c}_{2}$, so that $h_{2}\left(\gamma\left(\bar{c}_{1}\right)\right) \neq 0$.

Then, substituting for $R\left(c_{1}, \gamma\left(c_{1}\right)\right)=\left[2\left(a-c_{1}\right) \sqrt{b K}-b K\right] / 4 b$ and $h_{1}$ and rearranging terms yields

$$
\frac{d s^{*}}{d c}=\frac{\delta_{1} \rho}{\left[a-c_{1}-2 b s^{*}\left(c_{1}\right)\right]} .
$$

This differential equation was derived on the assumption that $s^{*}$ was monotone decreasing on $\left[\underline{c}_{1}, \bar{c}_{1}\right]$. The solutions meeting this condition and satisfying the non-negativity condition for expected profits form a non-intersecting family parameterized by a boundary condition, which we may take to be the value of $s^{*}\left(\bar{c}_{1}\right)$. Since each member of this family with the appropriate specification of $t^{*}$ 
can constitute an equilibrium, ${ }^{11}$ the multiplicity of equilibria in the earlier example carries over.

As suggested earlier, it seems reasonable to concentrate on solutions which are Pareto efficient. There is a unique such solution among the separating equilibria. In it, the highest cost firm, which will stand revealed as a weakling in any case, does not limit price. Alternatively, we can also eliminate the multiplicity by imposing the condition that an entrant whose costs exceed $\gamma\left(\bar{c}_{1}\right)$ will never enter, no matter what value of $Q$ is observed, since such an entrant could never expect to recoup the entry cost $K$. Under either of these specifications, the boundary condition becomes $s^{*}\left(\bar{c}_{1}\right)=m\left(\bar{c}_{1}\right)=\left(a-\bar{c}_{1}\right) / 2 b$. The corresponding solution of the differential equation is then given implicitly by

$$
0=m\left(c_{1}\right)-s^{*}\left(c_{1}\right)+\delta_{1} \rho-\delta_{1} \rho \exp \left[\frac{m\left(\bar{c}_{1}\right)-s^{*}\left(c_{1}\right)}{\delta_{1} \rho}\right] .
$$

Now, let $t^{*}$ be specified by $t\left(c_{2}, Q\right)=1$ iff $c_{2} \leqq \gamma\left(s^{*-1}(Q)\right)$ for $Q$ in the range of $s^{*}$ and, say, by $t\left(c_{2}, Q\right)=t\left(c_{2}, s^{*}\left(\underline{c}_{1}\right)\right)$ for $Q>s^{*}\left(\underline{c}_{1}\right)$ and $t\left(c_{2}, Q\right)=t\left(c_{2}\right.$, $\left.s^{*}\left(\bar{c}_{1}\right)\right)$ for $Q<m\left(\bar{c}_{1}\right)$. For $s^{*}$ and $t^{*}$ to be an equilibrium it is clearly sufficient that $G\left(c_{1}, Q\right)$ be pseudo-concave in $Q$ for each $c_{1}$, so that the first order condition (1) guarantees an optimum. For this, it is in turn sufficient (see [11]) that

$$
\begin{aligned}
\frac{d s^{*}(z)}{d z} \leqq & \inf _{c \in\left[0, \bar{c}_{1}\right]} \frac{\delta_{1} \gamma^{\prime}(z)\left[R\left(c_{1}, \gamma(z)\right)-R(z, \gamma(z))\right] h_{1}(\gamma(z))}{(z-c)} \\
= & \inf _{c_{1}} \frac{\delta_{1}(1 / 2)\left[\left(z-c_{1}\right)\left(24 \sqrt{b K}-6 a-z+7 c_{1}\right) / 36 b\right]}{\left(z-c_{1}\right)} \\
& \times\left(\frac{8 b \rho}{2(a-z) \sqrt{b K}-b K}\right)=\frac{\delta_{1} \rho[24 \sqrt{b K}-6 a-z]}{9[2(a-z) \sqrt{b K}-b K]} .
\end{aligned}
$$

Since $d s^{*} / d z=-1 /\left[2 b\left(1-\exp \left[\left(m\left(\bar{c}_{1}\right)-s(z)\right) / \delta_{1} \rho\right]\right)\right]$ is strictly decreasing and bounded above by $-1 /(2 b)$, if the right hand side of the inequality were always positive, i.e., $6 a+\sup z<24 \sqrt{b K}$, we would then be assured that $\left(s^{*}, t^{*}\right)$ is an equilibrium. Thus, since $\bar{c}_{1}=\sup z<a / 2, a<48 \sqrt{b K} / 13$ provides a sufficient condition.

It is straightforward to obtain comparative statics results for this example. Let $A \equiv\left[m\left(\bar{c}_{1}\right)-s\left(c_{1}\right)\right] /\left(\delta_{1} \rho\right) \leqq 0$. Then

$$
\begin{gathered}
\partial s^{*} / \partial \rho=\delta_{1} \frac{[1+(A-1) \exp A]}{1-\exp A}>0, \\
\partial s^{*} / \partial \delta_{1}=\rho \frac{[1+(A-1) \exp A]}{1-\exp A}>0,
\end{gathered}
$$

\footnotetext{
"So long as the first-order condition (1) actually gives a maximum.
} 
and

$$
\partial s^{*} / \partial \bar{c}_{1}=\frac{\exp A}{2 b(1-\exp A)}>0 .
$$

The intuition behind the first two results is clear. Regarding the third, the idea is that the possibility of there being higher cost firms leads the current $\bar{c}_{1}$ firm to limit price in order to distinguish itself, and then all lower cost firms must further increase their outputs.

Since the particular $h$ function that we chose to permit computation resulted in $R\left(c_{1}, \gamma\left(c_{1}\right)\right) h\left(\gamma\left(c_{1}\right)\right)$ being constant, comparative statics with respect to $a$ and $b$ reveal the effects of changes in first period demand only. Note too that changes in these parameters affect both $m$ and $s^{*}$, so interest centers on the effects on $s^{*}-m$. These are obtained by $\partial s^{*} / \partial a=1 / 2 b=\partial m / \partial a$, and $\partial s^{*} / \partial b<-$ $\left(a-c_{1}\right) / 2 b^{2}=\partial m / \partial b$ : increases in $a$ do not affect the amount of limit pricing, while increases in $b$ reduce the amount of limit pricing by increasing the marginal cost of this activity (as measured by $c_{1}$ less the marginal revenue at $s^{*}\left(c_{1}\right)$ ) while leaving the marginal return (in the second period) unaffected.

Since the density function we used depends on $K$, comparative statics with respect to $K$ cannot legitimately be interpreted in the natural way as indicating the effect of changing entry barriers. ${ }^{12}$ To allow such an analysis, suppose instead that the established firm's beliefs are given by a density function which is independent of $K$. In this case, if $K=0$, then $R\left(c_{1}, \gamma\left(c_{1}\right)\right) h\left(\gamma\left(c_{1}\right)\right) \equiv 0$, and no limit pricing will occur. It is only the fact of positive $K$ that causes the marginal entrant to enter with a strictly positive level of output. With no cost of entry, a marginal entrant comes in with an output which is essentially zero, and there is no return to deterring such entry. Similarly, if $K$ is very large (Bain's blockaded entry case), no possible level of $\underline{c}_{2}$ will permit positive profits, the threat of entry disappears, and again no limit pricing will occur. In the particular example we calculated, $K$ was such that $\gamma\left(\underline{c}_{1}\right)>\underline{c}_{2}$, so even low cost established firms were threatened and practiced limit pricing. A fourth possibility comes when $K$ is high enough that $\gamma\left(\underline{c}_{1}\right)<\underline{c}_{2}$, so that there is a set $\left[\underline{c}_{1}, c_{1}^{*}\right)$ of firms against which no potential entrant would want to enter. An interesting aspect of our model is that even firms in this range may practice limit pricing. The essential cause of this is that, if $m\left(c_{1}^{\prime}\right) \leqq s\left(c_{1}^{\prime \prime}\right)$ for some $c_{1}^{\prime}<c_{1}^{*}<c_{1}^{\prime \prime}$, then by producing $m\left(c_{1}^{\prime}\right)$, the low cost firm becomes identified with higher cost firms which are subject to entry. These latter firms may be expected to be limit pricing, so $s^{*}\left(c_{1}^{\prime \prime}\right)>m\left(c_{1}^{\prime \prime}\right)$, and thus $m\left(c_{1}^{\prime}\right)=s^{*}\left(c_{1}^{\prime \prime}\right)$ is possible. By increasing output to (slightly more than) $s^{*}\left(c_{1}^{*}\right)$, which, to a first approximation, does not reduce the value of $\Pi^{0}$, the low cost firm can eliminate the threat of entry and thus increase second period expected returns.

Finally, we should mention that although we have concentrated on separating equilibria, other equilibria are possible in the continuum of types framework. A

\footnotetext{
${ }^{12}$ The possibility of normalizing 2's payoff means that lowering $\delta_{2}$ corresponds to raising $K$.
} 
result of Milgrom and Weber [13] indicates that we need not concern ourselves with mixed strategy equilibria in games of this type. However, pure pooling equilibria are conceptually possible, as are equilibria where $s^{*}$ is a decreasing step function. ${ }^{13}$ In any pooling equilibrium, all types of the established firm are better off producing the equilibrium output $Q^{*}$ than they are changing their output and facing the different probability of entry this different value of $Q$ implies. For example, if entry is relatively unlikely when $Q=Q^{*}$ (perhaps because low values of $c_{1}$ are very likely a priori), and any deviation from $Q^{*}$ brings certain entry, then if the $\bar{c}_{1}$ type is willing to produce $Q^{*}$, a pooling equilibrium will be maintained. In general, the form of the entrant's conjectures (as embodied in its strategy) which is necessary to support a pooling equilibria is typically discontinuous in $Q$, and the same sort of discontinuities underlie step-function equilibria.

It is clear that the extended example we have been discussing involves a number of special features, such as the linearity of demand and cost, and the assumption that post-entry competition yields the full information Cournot outcome. However, these assumptions serve mainly to simplify arguments and facilitate computation; they do not drive the results. Indeed, so long as the entrant's post-entry profits decrease in $c_{2}$ and increase in $c_{1}$ while the established firm strictly prefers to be a monopolist than to share the market $\left(R\left(c_{1}, c_{2}\right)>0\right)$, our principal conclusions remain: if pre-entry price can be a signal for post-entry profits, even if it does not directly influence profitability, then limit pricing will emerge in equilibrium, but entry need not be deterred relative to the complete information case. Moreover, as we shall argue in the next section, even if we allow for much more general uncertainty and for post-entry profits being dependent on pre-entry actions, a similar conclusion is valid.

\section{ENTRY DETERRENCE AND RATIONAL EXPECTATIONS}

In this section we consider a fairly general two-period model of entry deterrence and entry under incomplete information. While we do not provide a complete analysis of this model, we do indicate some of the implications of equilibrium for the firms' behavior.

Rather than setting up a general formal model from scratch, let us re-interpret the model in Section 2 with some modifications. In particular, we now view $c_{1}$ and $c_{2}$ as belonging to some arbitrary measurable spaces, and we will view $Q$ as an action belonging to some other arbitrary space. Suppose further that 2 observes only some variable $q$ which is correlated with $Q$, and suppose, too, that the payoffs depend not only on $c_{1}, c_{2}$ and the action $y$ taken by the entrant (which may also now belong to some arbitrary space), but also on $Q$ and possibly on a random variable $\theta$, the realization of which is not revealed until the firms make their choices. Finally, let all the random variables have some arbitrary joint distribution.

\footnotetext{
${ }^{13}$ The possible equilibria are characterized in [11].
} 
This framework is obviously very general. In particular, it allows for capital investment which affects marginal costs, advertising and other means of achieving brand loyalty, general forms of demand and cost functions, varying scales and forms of entry, imperfect observability of actions, uncertainty as to how the post-entry game will be played, and arbitrary dependencies among all the random elements of the model.

As before, it is useful to analyze equilibrium via strategies, $s^{*}$ and $t^{*}$, and conjectures, $\bar{s}$ and $\bar{t}$. (These may be taken to be either pure or mixed strategies.) Thus, firm 1 conjectures that 2's strategy is $\bar{t}$, for each value of $c_{1}$ it will select an action $Q=s\left(c_{1}\right)$ to maximize the expected value of its perceived payoff, conditional on $c_{1}$. Unless expected second-period payoffs are insensitive to $Q$, both through any direct effect on second period profits and also through the effect on 2's conjectured action, the solution for the established firm's maximization problem will not be the same as the solution to the problem of maximizing the expected value of first period profits. Thus, we would generally expect that the threat of entry will alter behavior: some generalized form of limit pricing will be a characteristic of equilibrium.

In making its decision, the entrant will seek to maximize its expected payoff conditional on its private information $c_{2}$ and its (imperfect) observation of $Q$, given its conjecture $\bar{s}$. Should it happen that the observation of the signal $q$ in equilibrium permits a precise inference via $\bar{s}$ about $c_{1}$, then entry will of course occur in precisely the same circumstances as if $c_{1}$ had been directly announced. In this case, the only effect of the generalized limit pricing on entry will be through the direct effect of $Q$ on 2's post-entry profits (as, for example, when the choice of $Q$ affects demand or cost). If this effect is zero, then, as in the example in Section 2, limit pricing will still occur, but it need not deter entry relative to the complete information case.

However, the unrestricted dimensionalities allowed for $c_{1}$ and $Q$ suggest that an invertible strategy $s^{*}$ is unlikely. Moreover, so long as the random noise term relating $q$ and $Q$ is neither perfectly correlated with $c_{1}$ nor degenerate, then even if $s^{*}$ is an invertible function of $Q$ one would not expect a noisy observation of $Q$ via $q$ to permit a precise inference of the value of $c_{1}$. Thus one must expect that such exact inferences will be impossible in equilibrium, and that residual uncertainty will remain concerning $c_{1}$ when the entry decision is made. In this case, the entrant must base its entry decision $y$ on the expected value of its profits, as a function of $Q, y$ and the exogenous uncertainty $\theta$, conditional on the values of $c_{2}$ and $q$, and given its conjecture $\bar{s}$ about l's behavior. With some abuse of notation, let us write this as

$$
E\left(\Pi_{2}\left(c_{1}, c_{2}, \bar{s}\left(c_{1}\right), y, \theta\right) \mid q\left(\bar{s}\left(c_{1}\right), \theta\right), c_{2}\right) .
$$

Then the question is that of whether the established firm can, through its choice of $Q$, cause the entrant in equilibrium to lower its estimate of the profitability of entry. 
Consider what 2's estimate of its prospects are a priori, knowing $c_{2}$ but before observing $q$. This is just the expectation of expression (2), conditional on $c_{2}$. Then, in equilibrium, where $\bar{s}=s^{*}$, so that 2's conjecture is correct, this a priori estimate is

$$
E\left(E\left[\Pi_{2}\left(c_{1}, c_{2}, s^{*}\left(c_{1}\right), y, \theta\right) \mid q\left(s^{*}\left(c_{1}\right), \theta\right), c_{2}\right] \mid c_{2}\right) .
$$

But, by a standard result in probability theory, expression (3) is equal to $E\left(\Pi_{2}\left(c_{1}, c_{2}, s^{*}\left(c_{1}\right), y, \theta\right) \mid c_{2}\right)$. But this, in turn, is simply what firm 2 would estimate its profits to be if it were to receive no information.

In this sense, then, the observation of the established firm's actions cannot, in equilibrium, systematically bias the entrant's expectations. If without any information it would have estimated its expected profits at $\bar{\Pi}$ then the fact that it will receive the signal cannot lead it to expect to receive less than $\bar{\Pi}$. Put a different way, if there are some values of $c_{1}$ and $c_{2}$ such that observing $s^{*}\left(c_{1}\right)$ (directly or indirectly) causes an entrant with characteristics $c_{2}$ to underestimate the profitability of entry, then there is an offsetting set of values for $c_{1}$ and $c_{2}$ where observing $s^{*}\left(c_{1}\right)$ causes the entrant to overestimate its prospects.

\section{SUMMARY AND CONCLUSIONS}

In his original analysis of limit pricing, Bain [1, p. 453] argued that although "current price... need play no direct role [in the entry decision], since the anticipated industry price after entry and the entrant's anticipated market share are the strategic considerations," the potential entrant may "regard this price as an indicator" of post-entry profitability. Given this, Bain developed his theory of limit pricing, from which a large literature has emerged. A weakness of this literature has been the failure to model both the established firm and the entrant as strategic agents. However, if one models the situation described by Bain as a game of complete information, no limit pricing can emerge in equilibrium [3].

In this paper we model the problem considered by Bain of entry deterrence and entry as a game of incomplete information. In this game, Bain's arguments are valid: although pre-entry actions by the established firm may not influence post-entry profitability, they may become signals for some unobservable determinants of profits. Limit pricing, or, more generally, deviations from short run maximizing behavior, then emerge in equilibrium, just as earlier analyses had found. However, an unsuspected feature also emerges. Since the entrant will, in equilibrium, recognize the incentives for limit-pricing, its expectations of the profitability of entry will not be consistently biased by the established firm's behavior. Then, depending on the particular equilibrium that is established and the parameters of the model, the probability of entry may fall short of, equal, or even exceed what it would be if there were complete information and thus no limit pricing.

One conclusion of this analysis is for the appropriate public policy towards 
limit-pricing. If pre-entry price does not influence post-entry demand and if the two-period modelling used here is appropriate, then limit pricing should not be discouraged, since it means lower prices and cannot, overall, limit entry. More generally, the admittedly incomplete analysis in Section 3 might suggest a stronger statement regarding strategic moves taken by established firms to deter entry. To the extent that these actions are not objectionable per se, but rather are of potential concern only because of signalling effects which it is feared may deter entry, then they are in fact benign. The question is whether either of these suggestions would stand up under a full examination of a richer model. In particular, it would seem that embedding the opportunity for limit pricing in a multi-period model where predation is possible and where reputations are a factor would be an important extension of the present analysis. This is a problem we hope to address in future work.

\section{Northwestern University \\ and \\ Stanford University}

Manuscript received March, 1980; revision received December, 1980.

\section{REFERENCES}

[1] BaIN, J.: "A Note on Pricing in Monopoly and Oligopoly," American Economic Review, 39(1949), 448-464.

[2] Clark, J. M.: "Toward a Concept of Workable Competition," American Economic Review, 30(1940), 241-256.

[3] Frifidman, J.: "On Entry Preventing Behavior," in Applied Game Theory, ed. by S. J. Brams, A. Schotter, and G. Schwodiauer. Wurzburg, Vienna: Physica-Verlag, 1979, pp. 236-253.

[4] Cial-or, E.: "Limit Price Entry Prevention and its Impact on Potential Investors-A GameTheoretic Approach," Ph.D. Dissertation, Northwestern University, 1980.

[5] Gaskins, D.: "Dynamic Limit Pricing: Optimal Pricing Under Threat of Entry," Journal of Economic Theory, 2(1971), 306-322.

[6] Harsanyi, J. C.: "Games with Incomplete Information Played by 'Bayesian' Players," Parts I, II and III, Management Science, 14 (November, 1967; January, 1968; and March, 1968), $159-182,320-324$, and $486-502$.

[7] KaI.DOR, N.: "Market Imperfection and Excess Capacity," Economica, 2(1935), 33-50.

[8] Kamifn, M. I., and N. Schwartz: "Limit Pricing and Uncertain Entry," Econometrica, 39(1971), 441-454.

[9] KREPS, D., and R. WIL.SON: "On the Chain-Store Paradox and Predation: Reputation for Toughness," Discussion Paper 551, Graduate School of Business, Stanford University, 1980.

[10] - _ : "Sequential Equilibria," Discussion Paper 584, Graduate School of Business, Stanford University, 1980.

[11] Mil.grom, P., and J. Roberts: “Equilibrium Limit Pricing Doesn’t Limit Entry,” Discussion Paper 399R, Center for Mathematical Studies in Economics and Management Science, Northwestern University, 1980.

[12] - - : "Predation, Reputation, and Entry Deterrence," Discussion Paper 427, Center for Mathematical Studies in Economics and Management Science, Northwestern University, 1980.

[13] Milgrom, P., and R. Weber: "Distributional Strategies for Games with Incomplete Information," Discussion Paper 428, Center for Mathematical Studies in Economics and Management Science, Northwestern University, 1980.

[14] Myrisson, R.: "Refinements of the Nash Equilibrium Concept," International Journal of Game Theory, 7(1978), 73-80. 
[15] Ortega-Reichert, A.: "Models for Competitive Bidding Under Uncertainty," Ph.D. dissertation, Stanford University, 1968.

[16] Pyatt, G.: "Profit Maximization and the Threat of New Entry," Economic Journal, 81(1971), $242-255$.

[17] Salop, S. C.: "Strategic Entry Deterrence," American Economic Review, 69(1979), 335-338.

[18] Scherer, F. M.: Industrial Market Structure and Economic Performance, Second Edition. Chicago: Rand McNally and Company, 1979.

[19] Selten, R.: "Reexamination of the Perfectness Concept for Equilibrium Points in Extensive Games," International Journal of Game Theory, 4(1975), 25-55. 